\title{
ON HAUSDORFF DIMENSIONS RELATED TO SETS WITH GIVEN ASYMPTOTIC AND GAP DENSITIES
}

\author{
Ladislav Mišík — Jan Šustek — Bodo Volkmann \\ Dedicated to the memory of Professor Pierre Liardet
}

\begin{abstract}
For a set $A$ of positive integers $a_{1}<a_{2}<\cdots$, let $\underline{d}(A), \bar{d}(A)$ denote its lower and upper asymptotic densities. The gap density is defined as $\lambda(A)=\limsup _{n \rightarrow \infty} \frac{a_{n+1}}{a_{n}}$. The paper investigates the class $\mathcal{G}(\alpha, \beta, \gamma)$ of all sets $A$ with $\underline{d}(A)=\alpha, \bar{d}(A)=\beta$ and $\lambda(A)=\gamma$ for given $\alpha, \beta, \gamma$ with $0 \leq \alpha \leq \beta \leq 1 \leq \gamma$ and $\alpha \gamma \leq \beta$. Using the classical dyadic mapping $\varrho(A)=\sum_{n=1}^{\infty} \frac{\chi_{A}(n)}{2^{n}}$, where $\chi_{A}$ is the characteristic function of $A$, the main result of the paper states that the $\varrho$-image set $\varrho \mathcal{G}(\alpha, \beta, \gamma)$ has the Hausdorff dimension

$$
\operatorname{dim} \varrho \mathcal{G}(\alpha, \beta, \gamma)=\min \left\{\delta(\alpha), \delta(\beta), \frac{1}{\gamma} \max _{\sigma \in[\alpha \gamma, \beta]} \delta(\sigma)\right\},
$$
\end{abstract}

where $\delta$ is the entropy function

$$
\delta(x)=-x \log _{2} x-(1-x) \log _{2}(1-x) .
$$

Communicated by Vladimír Baláž

\section{Introduction}

Let $\mathcal{P}$ and $\mathcal{P}_{\infty}$, respectively, be the classes of all sets of positive integers and of all infinite sets of positive integers. Each $A \in \mathcal{P}_{\infty}$ can be mapped onto a real number $\varrho(A) \in(0,1]$ via the map

$$
\varrho(A)=\sum_{n=1}^{\infty} \frac{\chi_{A}(n)}{2^{n}},
$$

2010 Mathematics Subject Classification: 11B05, 11K55.

Keywords: Sequences of integers, lower asymptotic density, upper asymptotic density, gap density, Hausdorff dimension.

This work was supported by the European Regional Development Fund in the IT4Innovations Centre of Excellence project (CZ.1.05/1.1.00/02.0070), by the project P201/12/2351 of GAČR and by the grant 01798/2011/RRC of the the Moravian-Silesian Region. 


\section{LADISLAV MIŠÍK - JAN ŠUSTEK - BODO VOLKMANN}

$\chi_{A}$ being the characteristic function of $A$. The mapping is a bijection of $\mathcal{P}_{\infty}$ onto $(0,1]$. Having a class $\mathcal{A} \subset \mathcal{P}_{\infty}$, we will denote by

$$
\varrho \mathcal{A}=\{\varrho(A) \mid A \in \mathcal{A}\}
$$

the set of all images of elements of $\mathcal{A}$.

By log we will always mean the binary logarithm. Recall that the entropy function (of one variable) is the function defined on the interval $[0,1]$ by

$$
\delta(x)=-x \log x-(1-x) \log (1-x) \quad(0<x<1)
$$

and $\delta(0)=\delta(1)=0$, rendering the function continuous. It should be noted that the function $\delta$ is monotonically increasing from 0 to 1 in the interval $\left[0, \frac{1}{2}\right]$ and that $\delta(1-x)=\delta(x)$ for all $x \in[0,1]$.

For any set $A \in \mathcal{P}$ and any $n \in \mathbb{N}=\{1,2, \ldots\}$ we let

$$
A^{n}=A \cap\{1, \ldots, n\},
$$

calling $A^{n}$ the $n$th initial subset of $A$. Furthermore, we use the notation

$$
A(n)=\operatorname{card} A^{n}
$$

and

$$
A(x)=A(\lfloor x\rfloor) \quad \text { for real } x \geq 1 .
$$

By $\lfloor x\rfloor$ we mean the integer part of $x$.

We denote the lower and upper asymptotic density, respectively, by

$$
\underline{d}(A)=\liminf _{n \rightarrow \infty} \frac{A(n)}{n} \text { and } \bar{d}(A)=\limsup _{n \rightarrow \infty} \frac{A(n)}{n} .
$$

Now we recall the concept of Hausdorff dimension of sets of real numbers. Let $A \subset \mathbb{R}$. For $s \geq 0$ and $\varepsilon>0$ denote

$$
H_{\varepsilon}^{s}(A)=\inf \left\{\sum_{n=1}^{\infty}\left(b_{n}-a_{n}\right)^{s} \mid A \subset \bigcup_{n=1}^{\infty}\left(a_{n}, b_{n}\right) \text { and } b_{n}-a_{n}<\varepsilon \text { for all } n \in \mathbb{N}\right\} \text {. }
$$

Note that $H_{\varepsilon}^{s}(A)$ is decreasing with respect to $\varepsilon$ which yields that the limit

$$
H^{s}(A)=\lim _{\varepsilon \rightarrow 0+} H_{\varepsilon}^{s}(A)
$$

exists and is called the s-dimensional Hausdorff measure of $A$. It is an exercise (see, e.g., [1]) to show that there is a unique real number $D \in[0,1]$ such that

$$
H^{s}(A)=\infty \text { for all } s<D \text { and } H^{s}(A)=0 \text { for all } s>D .
$$

The number $D$ is called Hausdorff dimension of $A$ and is denoted by $\operatorname{dim} A$. 


\section{ON HAUSDORFF DIMENSIONS RELATED TO DENSITIES}

In [3], Theorem 52, it is proved, among other results, that given any $\alpha$ and $\beta$, $0 \leq \alpha \leq \beta \leq 1$, the set

$$
\mathcal{G}(\alpha, \beta)=\{A \in \mathcal{P} \mid \underline{d}(A)=\alpha, \bar{d}(A)=\beta\}
$$

has an image set $\varrho \mathcal{G}(\alpha, \beta)$ with Hausdorff dimension

$$
\operatorname{dim} \varrho \mathcal{G}(\alpha, \beta)=\min \{\delta(\alpha), \delta(\beta)\} .
$$

In this paper we investigate the sets $\mathcal{G}(\alpha, \beta)$ in more detail by introducing the gap density $\lambda(A)$ as an additional parameter. As defined in [2], given any set $A=\left\{a_{1}<a_{2}<\ldots\right\} \in \mathcal{P}_{\infty}$, we let

$$
\lambda(A)=\limsup _{n \rightarrow \infty} \frac{a_{n+1}}{a_{n}},
$$

noting that $\lambda(A)$ may be infinite. Obviously, the gap density is related to the lower and upper asymptotic densities by the inequality

$$
1 \leq \lambda(A) \leq \frac{\bar{d}(A)}{\underline{d}(A)} \quad \text { for all } A \in \mathcal{P}_{\infty} .
$$

For all $\alpha, \beta$, $\gamma$ with $0 \leq \alpha \leq \beta \leq 1$ and $1 \leq \gamma \leq \frac{\beta}{\alpha}$ (with $\frac{\beta}{0}=\infty$ ) we define the class

$$
\mathcal{G}(\alpha, \beta, \gamma)=\left\{A \in \mathcal{P}_{\infty} \mid \underline{d}(A)=\alpha, \bar{d}(A)=\beta, \lambda(A)=\gamma\right\} .
$$

The aim of the present paper is to determine the Hausdorff dimension of the set $\varrho \mathcal{G}(\alpha, \beta, \gamma)$.

\section{Homogeneous and saturated classes}

\subsection{Notation}

The following definitions and notation will be needed in our paper.

\subsection{1.}

For any class $\mathcal{S} \subset \mathcal{P}$ we let

$$
\mathcal{S}^{n}=\left\{A^{n} \mid A \in \mathcal{S}\right\} \quad(n=1,2, \ldots),
$$

noting that $\mathcal{S}^{n}$ is a class of finite sets. 


\section{LADISLAV MIŠÍK - JAN ŠUSTEK - BODO VOLKMANN}

\subsection{2.}

For any class $\mathcal{S} \subset \mathcal{P}$ and any pair $m, n$ with $m<n$, if $\mathcal{T} \subset \mathcal{S}^{m}$ is given, we define the class

$$
\mathcal{S}_{m}^{n}(\mathcal{T})=\bigcup_{T \in \mathcal{T}}\left\{S \in \mathcal{S}^{n} \mid S^{m}=T^{m}\right\}
$$

Hence $\mathcal{S}_{m}^{n}(\mathcal{T})$ is the class of all sets $S \in \mathcal{S}^{n}$ for which there exists a set $T \in \mathcal{T}$ having the same $m$ th initial subset. We will call these sets $S$ the descendants of $\mathcal{T}$ in $\mathcal{S}^{n}$. We will write $\mathcal{S}_{m}^{n}(T)$ instead of $\mathcal{S}_{m}^{n}(\{T\})$.

\subsection{3.}

We define, for every nonempty class $\mathcal{S} \subset \mathcal{P}$,

$$
\iota(\mathcal{S})=\liminf _{n \rightarrow \infty} \frac{\log \operatorname{card} \mathcal{S}^{n}}{n} .
$$

Clearly, we always have $0 \leq \iota(\mathcal{S}) \leq 1$.

\subsection{4.}

As usual, we consider the basic dyadic intervals of order $n$,

$$
\left(\frac{k}{2^{n}}, \frac{k+1}{2^{n}}\right] \quad\left(k=0, \ldots, 2^{n}-1\right),
$$

denoting the set of these $2^{n}$ intervals by $\mathcal{B}^{n}$ and letting

$$
\mathcal{B}=\bigcup_{n=1}^{\infty} \mathcal{B}^{n}
$$

stand for the set of all basic dyadic intervals.

\subsection{5.}

For $T \in \mathcal{P}_{\infty}$ and $n \in \mathbb{N}$ denote by

$$
B\left(T^{n}\right)=\left\{\varrho(U) \mid U \in \mathcal{P}_{\infty} \text { and } U^{n}=T^{n}\right\}=\left(\sum_{i=1}^{n} \frac{\chi_{T}(i)}{2^{i}}, \sum_{i=1}^{n} \frac{\chi_{T}(i)}{2^{i}}+\frac{1}{2^{n}}\right]
$$

the element of $\mathcal{B}^{n}$ containing $\varrho(T)$, being uniquely determined by the first $n$ binary digits of $T$. Similarly, for any $\mathcal{S} \subset \mathcal{P}_{\infty}$ and $n \in \mathbb{N}$ we let

$$
B\left(\mathcal{S}^{n}\right)=\bigcup_{T \in \mathcal{S}} B\left(T^{n}\right)
$$




\section{ON HAUSDORFF DIMENSIONS RELATED TO DENSITIES}

Consequently, for every $\mathcal{S} \subset \mathcal{P}_{\infty}$ and $n \in \mathbb{N}$, the relation $\varrho \mathcal{S} \subset B\left(\mathcal{S}^{n}\right)$ holds. Thus we have

$$
\varrho \mathcal{S} \subset \bigcap_{n=1}^{\infty} B\left(\mathcal{S}^{n}\right) .
$$

The classes $\mathcal{S}$ for which also the opposite inclusion holds will be of special interest.

\subsection{6.}

For a class $\mathcal{R}=\left\{R_{i} \mid i \in \mathcal{I}\right\}$ with $R_{i} \subset \mathbb{R}$ for every $i \in \mathcal{I}$ we will write, as usually,

$$
\bigcup \mathcal{R}=\bigcup_{i \in \mathcal{I}} R_{i} \text {. }
$$

We will say that a class $\mathcal{R}$ covers a set $A \subset \mathbb{R}$ if

\subsection{7.}

$$
A \subset \bigcup \mathcal{R}
$$

We will call a class $\mathcal{S} \subset \mathcal{P}_{\infty}$ saturated if $\varrho \mathcal{S}=\bigcap_{n=1}^{\infty} B\left(\mathcal{S}^{n}\right)$.

It will turn out that, for saturated classes $\mathcal{G}$, it is relatively simple to determine $\operatorname{dim} \varrho \mathcal{G}$. But unfortunately, our classes $\mathcal{G}(\alpha, \beta, \gamma)$ are not saturated. Indeed, since the asymptotic densities (lower, upper and gap density) of a set $A$ do not depend on its initial subsets, each set $\varrho \mathcal{G}(\alpha, \beta, \gamma)$ is everywhere dense in $[0,1]$. Therefore, we always have

\subsection{8.}

$$
B\left(\mathcal{S}^{n}\right)=(0,1] \quad \text { if } \mathcal{S}=\mathcal{G}(\alpha, \beta, \gamma)
$$

A class $\mathcal{S} \subset \mathcal{P}_{\infty}$ will be called homogeneous if there exist a positive integer $n_{0}$, a constant $\kappa>1$ and a nondecreasing function

such that

$$
\varphi:(0, \infty) \rightarrow(0, \infty)
$$

$$
\log \varphi(n)=o(n)
$$

and for all $m, n \in \mathbb{N}$ with $n_{0} \leq m<n \leq \kappa m$, the inequality

$$
\operatorname{card} \mathcal{S}_{m}^{n}(S) \leq \varphi(m) \operatorname{card} \mathcal{S}_{m}^{n}(T)
$$

holds for any choice of the sets $S, T \in \mathcal{S}^{m}$.

Roughly speaking, this means the following: Considering any two subintervals, $I$ and $J$, of $[0,1]$, the number of basic intervals of order $n$ containing elements from $\varrho \mathcal{S}$ within both of them, increases, as $n$ tends to infinity, with comparable speed. (For explicit details see (12) below.) 


\section{LADISLAV MIŠÍK - JAN ŠUSTEK - BODO VOLKMANN}

Example 1. Consider the system

$$
\mathcal{S}=\left\{A \in \mathcal{P}_{\infty} \mid \text { if } \frac{A\left(n_{0}\right)}{n_{0}}>\frac{1}{2} \text { for some } n_{0}, \text { then } n \in A \text { for all } n>n_{0}\right\} .
$$

This system is not homogeneous.

\subsection{Lemmas}

The first lemma of this section is well-known and its simple proof follows from Stirling's formula.

Lemma 1. For any $\alpha \in[0,1]$,

$$
\left(\begin{array}{c}
n \\
\lfloor\alpha n\rfloor
\end{array}\right)=2^{(\delta(\alpha)+o(1)) n} \quad \text { as } n \rightarrow \infty .
$$

We will also need a simple modification of the previous lemma.

Lemma 2. For any $\alpha, \beta \in[0,1]$ with $\alpha \leq \beta$ we have

$$
\sum_{j=\lfloor\alpha n\rfloor}^{\lfloor\beta n\rfloor}\left(\begin{array}{l}
n \\
j
\end{array}\right)=2^{(\omega+o(1)) n} \quad \text { as } n \rightarrow \infty
$$

where $\omega=\max _{\sigma \in[\alpha, \beta]} \delta(\sigma)$.

P r o of. In the case $\alpha \leq \frac{1}{2} \leq \beta$ the result follows from Lemma11and inequalities

$$
2^{\left(\delta\left(\frac{1}{2}\right)+o(1)\right) n}=\left(\begin{array}{c}
n \\
\lfloor n / 2\rfloor
\end{array}\right) \leq \sum_{j=\lfloor\alpha n\rfloor}^{\lfloor\beta n\rfloor}\left(\begin{array}{l}
n \\
j
\end{array}\right) \leq 2^{n} .
$$

In the case $\alpha \leq \beta<\frac{1}{2}$ the result follows from Lemma 1 and inequalities

$$
2^{(\delta(\beta)+o(1)) n}=\left(\begin{array}{c}
n \\
\lfloor\beta n\rfloor
\end{array}\right) \leq \sum_{j=\lfloor\alpha n\rfloor}^{\lfloor\beta n\rfloor}\left(\begin{array}{l}
n \\
j
\end{array}\right) \leq \beta n\left(\begin{array}{c}
n \\
\lfloor\beta n\rfloor
\end{array}\right)=2^{(\delta(\beta)+o(1)) n} .
$$

The case $\frac{1}{2}<\alpha \leq \beta$ is analogous.

Lemma 3. Let $\varphi:(0, \infty) \rightarrow(0, \infty)$ be a non-decreasing function such that (3) holds, and let $r, s$ with $0<r<s$ be real numbers. Then there exists a real number $q>0$ and an $n_{0} \in \mathbb{N}$ such that

$$
(1+q)^{k} n_{0}(s-(1+q) r)>\sum_{i=0}^{k-1} \log \varphi\left((1+q)^{i} n_{0}\right)+k+3
$$

holds for all $k \in \mathbb{N}_{0}$. The number $q$ can be chosen arbitrarily small and the number $n_{0}$ can be chosen arbitrarily large. 


\section{ON HAUSDORFF DIMENSIONS RELATED TO DENSITIES}

Pr o of. Choose $q>0$ such that $c=s-(1+q) r>0$, and $n_{0} \in \mathbb{N}$ such that

$$
n_{0}>\frac{3}{c} \text { and } \frac{\log \varphi\left(\nu_{k}\right)+1}{\nu_{k}}<q c,
$$

where $\nu_{k}=(1+q)^{k} n_{0}$ for every $k \in \mathbb{N}_{0}$. In this notation, denoting the left-hand and right-hand sides of (5) by $L(k)$ and $R(k)$, respectively, we have to prove that

$$
L(k)=c \nu_{k}>\sum_{i=0}^{k-1} \log \varphi\left(\nu_{i}\right)+k+3=R(k) .
$$

Evidently,

$$
L(0)=c n_{0}>3=R(0) \quad \text { by (6) } .
$$

Furthermore, for $k \in \mathbb{N}_{0}$, we have

$$
\Delta L(k)=L(k+1)-L(k)=c\left(\nu_{k+1}-\nu_{k}\right)=c q \nu_{k}
$$

and

$$
\Delta R(k)=R(k+1)-R(k)=1+\log \varphi\left(\nu_{k}\right) .
$$

The second relation in (6) implies $\Delta R(k)<\Delta L(k)$ for all $k \in \mathbb{N}_{0}$, which proves (5) by induction.

\section{Computation of dimensions}

Lemma 4. For each nonempty $\mathcal{S} \subset \mathcal{P}_{\infty}$,

$$
\operatorname{dim} \varrho \mathcal{S} \leq \iota(\mathcal{S}) .
$$

P r o of. It is sufficient to show that for any $r>\iota(\mathcal{S})$ and $\eta>0$ there exists a covering $\mathcal{J}$ of $\varrho \mathcal{S}$, consisting of countably many intervals $J$ with length $|J|<\eta$, and furthermore,

$$
\sum_{J \in \mathcal{J}}|J|^{r}<1
$$

To see this, first choose $n \in \mathbb{N}$ such that, in the sense of 2.1.1

$$
n>-\log \eta \text { and } \operatorname{card} \mathcal{S}^{n}<2^{r n} .
$$

Let $\mathcal{J}=\left\{B\left(T^{n}\right) \mid T \in \mathcal{S}\right\}$. Then $\mathcal{J}$ covers $\varrho \mathcal{S},|J|=2^{-n}<\eta$ for every $J \in \mathcal{J}$ and using (77), we obtain

$$
\sum_{J \in \mathcal{J}}|J|^{r}<2^{r n} 2^{-r n}=1,
$$

which proves the lemma. 


\section{LADISLAV MIŠÍK - JAN ŠUSTEK - BODO VOLKMANN}

Our next goal is to show that under some additional conditions the opposite inequality $\operatorname{dim} \varrho \mathcal{S} \geq \iota(\mathcal{S})$ also holds.

Theorem 1. Let a nonempty class $\mathcal{S} \subset \mathcal{P}_{\infty}$ be saturated and homogeneous. Then

$$
\operatorname{dim} \varrho \mathcal{S}=\iota(\mathcal{S}) .
$$

Pro of. 1. Let us define the set

$$
X=\left\{\frac{a}{2^{b}} \mid b \in \mathbb{N}, a=0, \ldots, 2^{b}\right\}
$$

of all dyadic rational points in $[0,1]$. By Lemma 4 it suffices to prove the inequality

$$
\operatorname{dim} \varrho \mathcal{S} \geq \iota(\mathcal{S})
$$

For technical reasons we are going to establish this at first for $\operatorname{dim}(\varrho \mathcal{S} \cup X)$ instead of $\operatorname{dim} \varrho \mathcal{S}$.

2. If $\iota(\mathcal{S})>0$, let $r<\iota(\mathcal{S})$ be an arbitrary positive real number. To prove Theorem 1 it is sufficient to find an $\eta>0$ such that no family $\mathcal{J}=\left(J_{n}\right)$ of intervals with $\left|J_{n}\right|<\eta$ for every $n \in \mathbb{N}$ and

covers $\varrho \mathcal{S} \cup X$.

$$
\sum_{i=1}^{\infty}\left|J_{i}\right|^{r}<1
$$

Choose $s \in(r, \iota(\mathcal{S}))$. Let $q>0$ and $n_{0} \in \mathbb{N}$ be numbers guaranteed by Lemma 3 with $1+q<\kappa\left(\kappa\right.$ being the number from 2.1.8) and with $n_{0}$ large enough such that

$$
\operatorname{card} \mathcal{S}^{n}>2^{s n}
$$

for every $n \geq n_{0}$.

Now let a family $\mathcal{J}$, satisfying (8) with $\eta=2^{-n_{0}}$, be fixed, and suppose that its elements $J_{1}, J_{2}, \ldots$ are numbered so that $\left|J_{1}\right| \geq\left|J_{2}\right| \geq \cdots$ We use the notation

$$
n_{k}=\left\lfloor(1+q)^{k} n_{0}\right\rfloor \quad(k=0,1,2, \ldots) .
$$

For each $k$ we define the symbols

$$
j_{k}=\max \left\{j \in \mathbb{N}|| J_{j} \mid \geq 2^{-n_{k}}\right\} \quad \text { and } \quad \mathcal{J}_{k}=\left\{J_{1}, J_{2}, \ldots, J_{j_{k}}\right\} .
$$

Then

$$
1>\sum_{i=1}^{j_{k}}\left|J_{i}\right|^{r} \geq j_{k} 2^{-r n_{k}},
$$

and thus we have, for each $k \in \mathbb{N}$,

$$
\operatorname{card} \mathcal{J}_{k}=j_{k}<2^{r n_{k}} .
$$




\section{ON HAUSDORFF DIMENSIONS RELATED TO DENSITIES}

3. We are going to prove that $\mathcal{J}$ cannot be an $\eta$-covering of the set $\varrho \mathcal{S} \cup X$. To do so we use the following procedure. For each index $k$ we show that there is a nonempty closed subset $V_{k}$ of $\varrho \mathcal{S} \cup X$ which is disjoint from all elements of $\mathcal{J}_{j_{k+1}}$ and satisfies $V_{k} \subset V_{k-1}$. Therefore, the intersection $\bigcap_{k=1}^{\infty} V_{k}$ is nonempty and disjoint from $\bigcup \mathcal{J}$.

In order to accomplish this we construct the sequence of systems of intervals,

$$
\mathcal{I}_{k}=\left\{\overline{B(S)} \mid S \in \mathcal{S}^{n_{k}}\right\} \quad(k=0,1,2, \ldots),
$$

where $\overline{B(S)}$ denotes the closure of the interval $B(S)$. Since there is a one-to-one correspondence between the elements of $\mathcal{S}^{n_{k}}$ and the elements of $\mathcal{I}_{k}$, we have

$$
\operatorname{card} \mathcal{I}_{k}=\operatorname{card} \mathcal{S}^{n_{k}} .
$$

Define the sequence of systems of intervals,

$$
\mathcal{U}_{k}=\left\{M \in \mathcal{I}_{k}\left(\mathcal{U}_{k-1}\right) \mid M \text { is disjoint from all elements of } \mathcal{J}_{k+1}\right\}
$$

$(k=0,1,2, \ldots)$. Here we let $\mathcal{I}_{0}\left(\mathcal{U}_{-1}\right)=\mathcal{I}_{0}$ and denote the system of those descendants (see 2.1.2) of $\mathcal{U}_{k-1}$ which are contained in $\mathcal{I}_{k}$ by

$$
\mathcal{I}_{k}\left(\mathcal{U}_{k-1}\right)=\left\{M \in \mathcal{I}_{k} \mid \text { there exists } U \in \mathcal{U}_{k-1} \text { such that } M \subset U\right\} .
$$

4. One can easily prove that the condition (4) implies, for any $\mathcal{T} \subset \mathcal{S}^{n_{k}}$, inequality

$$
\operatorname{card} \mathcal{S}_{n_{k}}^{n_{k+1}}(\mathcal{T}) \geq \frac{1}{\varphi\left(n_{k}\right)} \frac{\operatorname{card} \mathcal{T}}{\operatorname{card} \mathcal{S}^{n_{k}}} \operatorname{card} \mathcal{S}^{n_{k+1}}
$$

Now we prove by induction that

$$
\operatorname{card} \mathcal{U}_{k}>\frac{1}{2^{k+1} \prod_{i=0}^{k-1} \varphi\left(n_{i}\right)} \operatorname{card} \mathcal{I}_{k}
$$

Since every element of $\mathcal{I}_{0}$ has length $2^{-n_{0}}$ and every element of $\mathcal{J}_{1}$ has length less than $\eta=2^{-n_{0}}$, it follows that every element of $\mathcal{J}_{1}$ can overlap with at most two elements of $\mathcal{I}_{0}$. Inasmuch as inequality (5) with $k=0$ implies the relation

$$
\left(s n_{0}-1\right)-\left(r n_{1}+1\right) \geq s n_{0}-r n_{0}(1+q)-2>0,
$$

it follows from (9) and (10) that

$$
\operatorname{card} \mathcal{U}_{0} \geq \operatorname{card} \mathcal{I}_{0}-2 \operatorname{card} \mathcal{J}_{1}>\frac{1}{2} \operatorname{card} \mathcal{I}_{0}+2^{s n_{0}-1}-2^{r n_{1}+1}>\frac{1}{2} \operatorname{card} \mathcal{I}_{0},
$$

which proves (13) for $k=0$. 


\section{LADISLAV MIŠÍK - JAN ŠUSTEK - BODO VOLKMANN}

Assume (13) for some fixed $k \in \mathbb{N}_{0}$. Relations (11), (12) and (13) imply

$$
\operatorname{card} \mathcal{I}_{k+1}\left(\mathcal{U}_{k}\right) \geq \frac{1}{\varphi\left(n_{k}\right)} \frac{\operatorname{card} \mathcal{U}_{k}}{\operatorname{card} \mathcal{I}_{k}} \operatorname{card} \mathcal{I}_{k+1}>\frac{1}{2^{k+1} \prod_{i=0}^{k} \varphi\left(n_{i}\right)} \operatorname{card} \mathcal{I}_{k+1} .
$$

Since every element of $\mathcal{I}_{k+1}$ has length $2^{-n_{k+1}}$ and every element of $\mathcal{J}_{k+2} \backslash \mathcal{J}_{k+1}$ has length less than $2^{-n_{k+1}}$, it follows that every element of $\mathcal{J}_{k+2}$ can overlap with at most two elements of $\mathcal{I}_{k+1}\left(\mathcal{U}_{k}\right)$. Thus (9) and (10) imply

$$
\begin{aligned}
\operatorname{card} \mathcal{U}_{k+1} & \geq \operatorname{card} \mathcal{I}_{k+1}\left(\mathcal{U}_{k}\right)-2 \operatorname{card} \mathcal{J}_{k+2} \\
& >\frac{1}{2^{k+1} \prod_{i=0}^{k} \varphi\left(n_{i}\right)} \operatorname{card} \mathcal{I}_{k+1}-2^{r n_{k+2}+1} \\
& >\frac{1}{2^{k+2} \prod_{i=0}^{k} \varphi\left(n_{i}\right)} \operatorname{card} \mathcal{I}_{k+1}+\frac{1}{2^{k+2} \prod_{i=0}^{k} \varphi\left(n_{i}\right)} 2^{s n_{k+1}}-2^{r n_{k+2}+1} \\
& =\frac{1}{2^{k+2} \prod_{i=0}^{k} \varphi\left(n_{i}\right)} \operatorname{card} \mathcal{I}_{k+1}+2^{s n_{k+1}-k-2-\sum_{i=0}^{k} \log \varphi\left(n_{i}\right)}-2^{r n_{k+2}+1} \\
& >\frac{1}{2^{k+2} \prod_{i=0}^{k} \varphi\left(n_{i}\right)} \operatorname{card} \mathcal{I}_{k+1} .
\end{aligned}
$$

For the last inequality we have used (5) for $k+1$ if $k$ is the index under consideration. Indeed,

$$
\begin{aligned}
& \left(s n_{k+1}-k-2-\sum_{i=0}^{k} \log \varphi\left(n_{i}\right)\right)-\left(r n_{k+2}+1\right) \\
& =\left\lfloor(1+q)^{k+1} n_{0}\right\rfloor s-\left\lfloor(1+q)^{k+2} n_{0}\right\rfloor r-\sum_{i=0}^{k} \log \varphi\left(\left\lfloor(1+q)^{i} n_{0}\right\rfloor\right)-k-3 \\
& >(1+q)^{k+1} n_{0} s-s-(1+q)^{k+2} n_{0} r-\sum_{i=0}^{k} \log \varphi\left((1+q)^{i} n_{0}\right)-k-3 \\
& =(1+q)^{k+1} n_{0}(s-(1+q) r)-\sum_{i=0}^{k} \log \varphi\left((1+q)^{i} n_{0}\right)-(k+1)-3+(1-s) \\
& >1-s>0 .
\end{aligned}
$$




\section{ON HAUSDORFF DIMENSIONS RELATED TO DENSITIES}

This finishes the inductive proof of (13).

5. The system $\mathcal{S}$ being saturated by assumption, we have

$$
\bigcap_{n=1}^{\infty} \bigcup_{S \in \mathcal{S}^{n}} B(S)=\varrho \mathcal{S} .
$$

This and (13) imply that the sets

$$
V_{k}=\bigcup \mathcal{U}_{k} \subset \varrho \mathcal{S} \cup X \quad(k=1,2, \ldots)
$$

form a decreasing sequence of nonempty closed sets in the compact $[0,1]$. Hence $V=\bigcap_{k=1}^{\infty} V_{k}$ is nonempty. In view of the definition of the classes $\mathcal{U}_{k}$ and the relation (14) we have

$$
\emptyset \neq V \subset(\varrho \mathcal{S} \cup X) \backslash \bigcup \mathcal{J}
$$

Thus

$$
\operatorname{dim}(\varrho \mathcal{S} \cup X) \geq r \quad \text { for every } \quad r<\iota(\mathcal{S})
$$

and hence, since the set $X$ is countable,

$$
\operatorname{dim} \varrho \mathcal{S}=\operatorname{dim}(\varrho \mathcal{S} \cup X) \geq \iota(\mathcal{S}),
$$

which finishes the proof of Theorem 1 .

Example 2. Let $A \in \mathcal{P}_{\infty}$. We will show that the system

$$
\mathcal{S}=\left\{B \in \mathcal{P}_{\infty} \mid B \subset A\right\}
$$

is homogeneous and saturated. The homogeneity follows immediately from the fact that for any $m<n$ and for every pair $S, T \in \mathcal{S}^{m}$ we have

$$
\operatorname{card} \mathcal{S}_{m}^{n}(S)=\operatorname{card} \mathcal{S}_{m}^{n}(T)=2^{\operatorname{card}(A \cap\{m+1, m+2, \ldots, n\})} .
$$

To prove that $\mathcal{S}$ is saturated, choose any $x \notin \bigcap_{n=1}^{\infty} B\left(\mathcal{S}^{n}\right)$. Then there is $n \in \mathbb{N}$ such that $x \notin B\left(\mathcal{S}^{n}\right)$. But this means that $\varrho^{-1}(x)$ is not a subset of $A$, consequently $x \notin \varrho \mathcal{S}$.

Now the application of Theorem 1 implies that

$$
\operatorname{dim} \varrho \mathcal{S}=\liminf _{n \rightarrow \infty} \frac{\log \operatorname{card} \mathcal{S}^{n}}{n}=\liminf _{n \rightarrow \infty} \frac{\log 2^{A(n)}}{n}=\underline{d}(A) .
$$

This result was first proved in [4]. 


\section{LADISLAV MIŠÍK - JAN ŠUSTEK - BODO VOLKMANN}

\section{Main result}

The main theorem of this paper is the following. (For definitions see the introduction.)

TheOREM 2. Let $\alpha, \beta, \gamma$ with $0 \leq \alpha \leq \beta \leq 1 \leq \gamma$ and $\alpha \gamma \leq \beta$ be given. Then

$$
\operatorname{dim} \varrho \mathcal{G}(\alpha, \beta, \gamma)=\min \left\{\delta(\alpha), \delta(\beta), \frac{1}{\gamma} \max _{\sigma \in[\alpha \gamma, \beta]} \delta(\sigma)\right\} .
$$

The result can be stated more explicitely in the following way.

$$
\operatorname{dim} \varrho \mathcal{G}(\alpha, \beta, \gamma)= \begin{cases}\min \left\{\delta(\alpha), \frac{\delta(\beta)}{\gamma}\right\} & \text { if } \alpha \gamma \leq \beta \leq \frac{1}{2} \\ \min \left\{\delta(\alpha), \delta(\beta), \frac{1}{\gamma}\right\} & \text { if } \alpha \gamma \leq \frac{1}{2} \leq \beta \\ \min \left\{\delta(\beta), \frac{1}{\gamma} \delta(\alpha \gamma)\right\} & \text { if } \frac{1}{2} \leq \alpha \gamma \leq \beta\end{cases}
$$

Before proving the main theorem we construct a system of auxiliary sets inductively. First we note that, using (2) and the fact that $\mathcal{G}(\alpha, \beta, \gamma) \subset \mathcal{G}(\alpha, \beta)$, it follows immediately that the theorem holds if either $\alpha=0$ or $\beta=1$. Thus we assume $0<\alpha$ and $\beta<1$ in the sequel, so having $1 \leq \gamma<\infty$.

We will use the special classes $\mathcal{Z}(\alpha, \beta, \gamma, \sigma) \subset \mathcal{P}_{\infty}$ defined as follows. Let $\alpha, \beta, \gamma, \sigma$ be real numbers with $0<\alpha \leq \alpha \gamma \leq \sigma \leq \beta<1$. Construct the sequences

$$
\begin{array}{rlrlrl}
a_{1} & =1, & b_{1} & =2, & c_{1} & =\left\lfloor\gamma b_{1}\right\rfloor, \\
a_{m} & =(m+1) d_{m-1}, & b_{m} & =(m+1) a_{m}, & =2 c_{1}, \\
c_{m} & =\left\lfloor\gamma b_{m}\right\rfloor, & d_{m} & =(m+1) c_{m}
\end{array}
$$

$(m=2,3, \ldots)$. Let $\mathcal{Z}(\alpha, \beta, \gamma, \sigma)$ denote the class of all sets $A \in \mathcal{P}_{\infty}$ such that, for every $m \in \mathbb{N}$,

$$
\begin{array}{rlrl}
\operatorname{card}\left(A \cap\left(d_{m-1}+(k-1) m, d_{m-1}+k m\right]\right) & =\lfloor\alpha m\rfloor, & & k=1, \ldots, d_{m-1}, \\
\operatorname{card}\left(A \cap\left(a_{m}+(k-1) m, a_{m}+k m\right]\right) & =\lfloor\sigma m\rfloor, & & k=1, \ldots, a_{m}, \\
A \cap\left(b_{m}, c_{m}\right\rfloor & =\emptyset &
\end{array}
$$

and

$$
\operatorname{card}\left(A \cap\left(c_{m}+(k-1) m, c_{m}+k m\right]\right)=\lfloor\beta m\rfloor, \quad k=1, \ldots, c_{m} .
$$

We call $W$ the set of all endpoints of intervals mentioned in these equations.

Lemma 5. All classes $\mathcal{Z}(\alpha, \beta, \gamma, \sigma)$ are homogeneous and saturated; moreover,

$$
\operatorname{dim} \varrho \mathcal{Z}(\alpha, \beta, \gamma, \sigma)=\min \left\{\delta(\alpha), \delta(\beta), \frac{\delta(\sigma)}{\gamma}\right\} .
$$




\section{ON HAUSDORFF DIMENSIONS RELATED TO DENSITIES}

Proof. 1. We consider $\alpha, \beta, \gamma, \sigma$ to be fixed, writing $\mathcal{Z}(\alpha, \beta, \gamma, \sigma)=\mathcal{Z}$. The cardinality of the class $\mathcal{Z}^{d_{m-1}}$ satisfies

$$
1 \leq \operatorname{card} \mathcal{Z}^{d_{m-1}} \leq 2^{d_{m-1}},
$$

hence we have, as $m \rightarrow \infty$,

$$
\operatorname{card} \mathcal{Z}^{d_{m-1}}=2^{o\left(a_{m}\right)} .
$$

Lemma 1 implies that

$$
\operatorname{card} \mathcal{Z}^{a_{m}}=\operatorname{card} \mathcal{Z}^{d_{m-1}} \cdot\left(\begin{array}{c}
m \\
\lfloor\alpha m\rfloor
\end{array}\right)^{d_{m-1}}=2^{o\left(a_{m}\right)+(\delta(\alpha)+o(1)) m d_{m-1}}
$$

and

$$
\lim _{m \rightarrow \infty} \frac{\log \operatorname{card} \mathcal{Z}^{a_{m}}}{a_{m}}=\lim _{m \rightarrow \infty}\left(\frac{o\left(a_{m}\right)}{a_{m}}+(\delta(\alpha)+o(1)) \frac{m d_{m-1}}{a_{m}}\right)=\delta(\alpha) .
$$

Similarly,

$$
\operatorname{card} \mathcal{Z}^{b_{m}}=\operatorname{card} \mathcal{Z}^{a_{m}} \cdot\left(\begin{array}{c}
m \\
\lfloor\sigma m\rfloor
\end{array}\right)^{a_{m}}=2^{o\left(b_{m}\right)+(\delta(\sigma)+o(1)) m a_{m}}
$$

and

$$
\lim _{m \rightarrow \infty} \frac{\log \operatorname{card} \mathcal{Z}^{b_{m}}}{b_{m}} \lim _{m \rightarrow \infty}\left(\frac{o\left(b_{m}\right)}{b_{m}}+(\delta(\sigma)+o(1)) \frac{m a_{m}}{b_{m}}\right)=\delta(\sigma) .
$$

One easily obtains the relation

$$
\lim _{m \rightarrow \infty} \frac{\log \operatorname{card} \mathcal{Z}^{c_{m}}}{c_{m}}=\lim _{m \rightarrow \infty} \frac{\log \operatorname{card} \mathcal{Z}^{b_{m}}}{\left\lfloor\gamma b_{m}\right\rfloor}=\frac{\delta(\sigma)}{\gamma} .
$$

Finally, we have

$$
\lim _{m \rightarrow \infty} \frac{\log \operatorname{card} \mathcal{Z}^{d_{m}}}{d_{m}}=\delta(\beta)
$$

Let

$$
F(n)=\frac{\log \operatorname{card} \mathcal{Z}^{n}}{n}
$$

If restricted to the elements $n$ of $W$, the function $F(n)$ is monotone in each of the intervals $\left[d_{m-1}, a_{m}\right],\left[a_{m}, b_{m}\right],\left[b_{m}, c_{m}\right]$ and $\left[c_{m}, d_{m}\right](m=2,3, \ldots)$. 


\section{LADISLAV MIŠíK - JAN ŠUSTEK - BODO VOLKMANN}

Therefore, it suffices to consider the points $a_{m}, b_{m}, c_{m}, d_{m}$ in order to determine the $\liminf _{n \rightarrow \infty} F(n)$. We obtain

$$
\begin{aligned}
\iota(\mathcal{Z})=\liminf _{n \rightarrow \infty} \frac{\log \operatorname{card} \mathcal{Z}^{n}}{n} & =\min \left\{\delta(\alpha), \delta(\sigma), \frac{\delta(\sigma)}{\gamma}, \delta(\beta)\right\} \\
& =\min \left\{\delta(\alpha), \delta(\beta), \frac{\delta(\sigma)}{\gamma}\right\} .
\end{aligned}
$$

2. Now we prove that the class $\mathcal{Z}$ is homogeneous. Choose positive sufficiently large integers $m, n$ such that $m<n<2 m$. Then there is a uniquely determined integer $k$ such that $n \in\left(d_{k-1}, d_{k}\right]$. The construction of the sequence $\left(d_{k}\right)$ implies $n \geq(k !)^{3}$, and consequently,

$$
k \leq \frac{1}{3} \log n<\log m .
$$

Now look at the construction of the class $\mathcal{Z}$. Each of the intervals $\left(d_{k-1}, a_{k}\right]$, $\left(a_{k}, b_{k}\right]$ and $\left(c_{k}, d_{k}\right]$ consists of many small blocks of $k$ elements. The number card $\mathcal{Z}^{p}$ increases in each such block by a factor $\left(\begin{array}{c}k \\ \lfloor\tau k\rfloor\end{array}\right)$, where $\tau$ is one of the real numbers $\alpha, \sigma, \beta$. Choose $S, T \in \mathcal{Z}^{m}$ arbitrarily. Then, letting an integer $p$ increase from $m$ to $n$, both numbers card $\mathcal{Z}_{m}^{p}(S)$ and card $\mathcal{Z}_{m}^{p}(T)$ increase by the same factor in each small block, except the first one (containing $m$ ). Their increase may differ at most by the factor $2^{k}$. Thus

$$
\operatorname{card} \mathcal{Z}_{m}^{n}(S) \leq \operatorname{card} \mathcal{Z}_{m}^{n}(T) 2^{k} \leq \operatorname{card} \mathcal{Z}_{m}^{n}(T) 2^{\log m}=\operatorname{card} \mathcal{Z}_{m}^{n}(T) m .
$$

Consequently, the class $\mathcal{Z}$ satisfies the definition of being homogeneous with a suitable $n_{0}, \kappa=2$ and $\varphi(m)=m$.

3. We finish the proof of the lemma by showing that the class $\mathcal{Z}$ is saturated. To do so, it is sufficient to show that for each $\xi \in(0,1] \backslash \varrho \mathcal{Z}$ there exists a positive integer $n$ such that $\xi \notin B\left(\mathcal{Z}^{n}\right)$. Choose an arbitrary $S \in \mathcal{Z}$. Then a set $T \in \mathcal{P}_{\infty}$ belongs to $\mathcal{Z}$ if and only if

$$
\operatorname{card} T^{n}=\operatorname{card} S^{n} \quad \text { holds for all } n \in W .
$$

Choose a real number $\xi \in(0,1] \backslash \varrho \mathcal{Z}$ and let $Y=\varrho^{-1}(\xi)$. Then there exists an integer $n \in W$ so that card $Y^{n} \neq \operatorname{card} S^{n}$. Consequently $\xi \notin B\left(\mathcal{Z}^{n}\right)$ and thus $\mathcal{Z}$ is saturated.

Proof of Theorem 2. First we use Lemma 5 to establish a lower bound for $\operatorname{dim} \varrho \mathcal{G}(\alpha, \beta, \gamma)$. For every $\sigma \in[\alpha \gamma, \beta]$ and $A \in \mathcal{Z}(\alpha, \beta, \gamma, \sigma)$ we have

$$
\underline{d}(A)=\alpha, \quad \bar{d}(A)=\beta \quad \text { and } \quad \lambda(A)=\gamma .
$$




\section{ON HAUSDORFF DIMENSIONS RELATED TO DENSITIES}

Therefore, $\mathcal{Z}(\alpha, \beta, \gamma, \sigma) \subset \mathcal{G}(\alpha, \beta, \gamma)$ and

$\operatorname{dim} \varrho \mathcal{G}(\alpha, \beta, \gamma) \geq \operatorname{dim} \varrho \mathcal{Z}(\alpha, \beta, \gamma, \sigma)=\min \left\{\delta(\alpha), \delta(\beta), \frac{\delta(\sigma)}{\gamma}\right\}$.

This holds for every $\sigma \in[\alpha \gamma, \beta]$, hence

$$
\operatorname{dim} \varrho \mathcal{G}(\alpha, \beta, \gamma) \geq \min \left\{\delta(\alpha), \delta(\beta), \frac{1}{\gamma} \max _{\sigma \in[\alpha \gamma, \beta]} \delta(\sigma)\right\} .
$$

Now we prove the opposite inequality to (15). Since the relation

$$
\operatorname{dim} \varrho \mathcal{G}(\alpha, \beta, \gamma) \leq \min \{\delta(\alpha), \delta(\beta)\}
$$

follows from (2), it is sufficient to show that, if $0<\alpha \leq \alpha \gamma \leq \beta<1$, then

$$
\operatorname{dim} \varrho \mathcal{G}(\alpha, \beta, \gamma) \leq \frac{1}{\gamma} \max _{\sigma \in[\alpha \gamma, \beta]} \delta(\sigma)=\mu .
$$

Properties of the function $\delta$, the assertion (2) and the relation

$$
\mathcal{G}(\alpha, \beta, \gamma) \subset \mathcal{G}(\alpha, \beta)
$$

imply that this clearly holds in the case $\gamma=1$. Thus we assume $\gamma>1$ in the rest of the proof.

To establish (16) it is sufficient to prove that, for each $r \in(\mu, 1)$, there exists a positive constant $C(r)$ such that, for every $\eta>0$, there exists a sequence $\left(J_{n}\right)$ of intervals such that $\left|J_{n}\right|<\eta$ for every $n \in \mathbb{N}$, satisfying

$$
\varrho \mathcal{G}(\alpha, \beta, \gamma) \subset \bigcup_{n=1}^{\infty} J_{n} \text { and } \sum_{n=1}^{\infty}\left|J_{n}\right|^{r}<C(r) .
$$

So let $r \in(\mu, 1)$ be given. Choose the real numbers

$$
s \in(\mu, r), \quad \alpha^{\prime} \in(0, \alpha), \quad \beta^{\prime} \in(\beta, 1), \quad \gamma^{\prime} \in(1, \gamma) \quad \text { and } \quad q>0
$$

such that $1+q<\gamma^{\prime}$,

and

$$
\frac{q}{1+q}-r+s<0
$$

$$
\max _{\sigma \in\left[\frac{\alpha^{\prime} \gamma^{\prime}}{1+q}, \beta^{\prime}+\frac{q}{1+q}\right]} \delta(\sigma)<\gamma^{\prime} s
$$

The possibility of this choice is guaranteed by the definition of $\mu$ and the continuity of the function $\delta$.

Now letting

$$
C(r)=\sum_{k=1}^{\infty} 2^{2+\left(\frac{q}{1+q}-r+s\right)(1+q)^{k}}
$$




\section{LADISLAV MIŠÍK - JAN ŠUSTEK - BODO VOLKMANN}

we see by (17) that $C(r)<\infty$. Let $\eta>0$ be given. Take $n_{0}>-\log \eta$. For each $A \in \mathcal{G}(\alpha, \beta, \gamma)$ denote by $n_{A} \geq n_{0}$ the least integer $n$ such that

$$
A \cap\left(n, \gamma^{\prime} n\right]=\emptyset \quad \text { and } \quad \alpha^{\prime} \gamma^{\prime}<\frac{A(n)}{n}<\beta^{\prime}
$$

For each $k \in \mathbb{N}$ we let $\nu_{k}=n_{0}(1+q)^{k}$ and

$$
\mathcal{G}_{k}=\left\{A \in \mathcal{G}(\alpha, \beta, \gamma) \mid \gamma^{\prime} n_{A} \in\left[\nu_{k-1}, \nu_{k}\right)\right\} .
$$

Note that $\mathcal{G}(\alpha, \beta, \gamma)=\bigcup_{k=1}^{\infty} \mathcal{G}_{k}$. By definition there exists, for each set $A \in \mathcal{G}_{k}$, an integer

such that

$$
m=n_{A} \in\left[\frac{\nu_{k-1}}{\gamma^{\prime}}, \frac{\nu_{k}}{\gamma^{\prime}}\right)
$$

$$
\alpha^{\prime} \gamma^{\prime} m<A(m)<\beta^{\prime} m .
$$

Consequently, in the sense of (1),

$\alpha^{\prime} \gamma^{\prime} \frac{\nu_{k-1}}{\gamma^{\prime}} \leq \alpha^{\prime} \gamma^{\prime} m<A(m)=A\left(\frac{\nu_{k}}{\gamma^{\prime}}\right) \leq A(m)+\left(\frac{\nu_{k}}{\gamma^{\prime}}-m\right)<\beta^{\prime} m+\frac{\nu_{k}}{\gamma^{\prime}}-m$.

Dividing by $\frac{\nu_{k}}{\gamma^{\prime}}$ we obtain

$$
\frac{\alpha^{\prime} \gamma^{\prime} \frac{\nu_{k-1}}{\gamma^{\prime}}}{\frac{\nu_{k}}{\gamma^{\prime}}}=\frac{\alpha^{\prime} \gamma^{\prime}}{1+q}<\frac{A\left(\frac{\nu_{k}}{\gamma^{\prime}}\right)}{\frac{\nu_{k}}{\gamma^{\prime}}}<\frac{\beta^{\prime} \frac{\nu_{k}}{\gamma^{\prime}}+\frac{\nu_{k-1}}{\gamma^{\prime}} q}{\frac{\nu_{k}}{\gamma^{\prime}}}=\beta^{\prime}+\frac{q}{1+q} .
$$

Hence

$$
A\left(\frac{\nu_{k}}{\gamma^{\prime}}\right)=\sigma_{A, k} \quad \text { for some } \sigma_{A, k} \in\left(\frac{\alpha^{\prime} \gamma^{\prime}}{1+q}, \beta^{\prime}+\frac{q}{1+q}\right) .
$$

This yields, using (18) and Lemma 2,

$$
\operatorname{card} \mathcal{G}_{k}^{\left\lfloor\frac{\nu_{k}}{\left.\gamma^{\prime}\right\rfloor}\right.} \leq 2^{\gamma^{\prime} s \frac{\nu_{k}}{\gamma^{\prime}}}=2^{s \nu_{k}}
$$

First relation in (19) with (20) imply that for every $A \in \mathcal{G}_{k}$ we have

Then (21) implies that

$$
A \cap\left(\frac{\nu_{k}}{\gamma^{\prime}}, \nu_{k-1}\right]=\emptyset \text {. }
$$

$$
\operatorname{card} \mathcal{G}_{k}^{\left\lfloor\nu_{k}\right\rfloor} \leq 2^{\left\lfloor\nu_{k}\right\rfloor-\left\lfloor\nu_{k-1}\right\rfloor} \operatorname{card} \mathcal{G}_{k}^{\left\lfloor\frac{\nu_{k}}{\gamma^{\prime}}\right\rfloor} \leq 2^{\nu_{k}-\nu_{k-1}+1} 2^{s \nu_{k}}=2^{1+\left(s+\frac{q}{1+q}\right) \nu_{k}} .
$$

Thus we can cover the set $\varrho \mathcal{G}_{k}$ by at most $2^{1+\left(s+\frac{q}{1+q}\right) \nu_{k}}$ intervals of length $2^{-\left\lfloor\nu_{k}\right\rfloor}$ forming a family $\mathcal{J}_{k}$. Hence we have

$$
\sum_{J \in \mathcal{J}_{k}}|J|^{r} \leq 2^{1+\left(s+\frac{q}{1+q}\right) \nu_{k}} 2^{-r\left\lfloor\nu_{k}\right\rfloor} \leq 2^{2+\left(\frac{q}{1+q}-r+s\right) n_{0}(1+q)^{k}} .
$$




\section{ON HAUSDORFF DIMENSIONS RELATED TO DENSITIES}

The family $\mathcal{J}=\bigcup_{k=1}^{\infty} \mathcal{J}_{k}$ covers $\varrho \mathcal{G}(\alpha, \beta, \gamma)$ and satisfies the inequality

$$
\sum_{J \in \mathcal{J}}|J|^{r} \leq \sum_{k=1}^{\infty} 2^{2+\left(\frac{q}{1+q}-r+s\right) n_{0}(1+q)^{k}}<C(r) .
$$

This implies (16) and together with (15) finishes the proof.

\section{REFERENCES}

[1] BERNIK, V. I.-DODSON, M. M.: Metric Diophantine A Approximation on Manifolds. Cambridge Univ. Press, Cambridge, 1999.

[2] GREKOS, G.-VOLKMANN, B.: On densities and gaps. Journal of Number Theory 26 (1987), 129-148.

[3] VOLKMANN, B.: Über Klassen von Mengen natürlicher Zahlen. J. Reine Angew. Math. 190 (1952), 199-230.

[4] VOLKMANN, B.: Über Hausdorffsche Dimensionen von Mengen, die durch Zifferneigenschaften charakterisiert sind I. Math. Zeits. 58 (1953), 284-287.

Received July 20, 2012

Accepted April 10, 2015

\section{Ladislav Mišik}

Department of Mathematics

University of Ostrava 83

Centre of Excellence IT4Innovations-

-Division UO-IRAFM

30. dubna 22

70103 Ostrava 1

CZECH REPUBLIC

E-mail: ladislav.misik@osu.cz

Jan Šustek

Department of Mathematics

University of Ostrava

30. dubna 22

Ostrava, 70103 ,

CZECH REPUBLIC

E-mail: jan.sustek@osu.cz

\section{Bodo Volkmann}

Postfach 1108

D-71692 Möglingen, GERMANY

E-mail: bodo.volkmann@t-online.de 